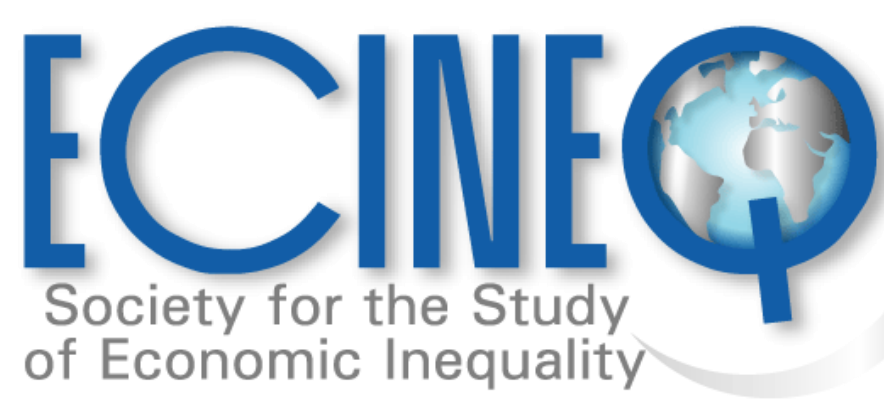

Working Paper Series

Conditions for the most robust multidimensional poverty comparisons using counting measures and ordinal variables

Gaston Yalonetzky 


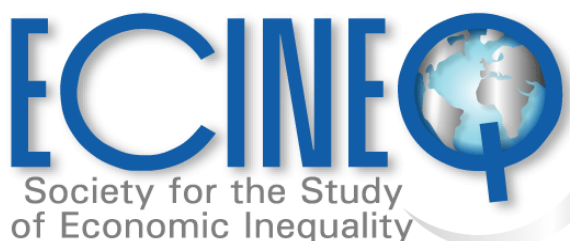

\title{
ECINEQ $2012-257$
}

June 2012

WWW.ecineq.org

\section{Conditions for the most robust multidimensional poverty comparisons using counting measures and ordinal variables*}

\author{
Gaston Yalonetzky \\ University of Leeds \& OPHI
}

\begin{abstract}
A natural concern with multivariate poverty measures, as well as with other composite indices, is the robustness of their ordinal comparisons to changes in the indices' parameter values. Applying multivariate stochastic dominance techniques, this paper derives the distributional conditions under which a multidimensional poverty comparison based on the popular counting measures, and ordinal variables, is fully robust to any values of the indices.parameters. As the paper shows, the conditions are relevant to most of the multidimensional poverty indices in the literature, including the Alkire-Foster family, upon which the UNDP.s "Multidimensional Poverty Index" (MPI) is based. The conditions are illustrated with an example from the EU-SILC dataset.
\end{abstract}

Keywords: Multidimensional poverty; stochastic dominance.

JEL Classification: I32.

\footnotetext{
* This version: 3 July 2012. I would like to thank Casilda Lasso de la Vega, Suman Seth, Jose Manuel Roche, Sabina Alkire, Julie Lichtfield, Paul Segal and seminar participants at the at the University of the Basque Country, Maastricth School of Governance, OPHI, and the XVI World Congress of the International Economic Association for helpful comments.

† Contact details: G.Yalonetzky@1eeds.ac.uk .
} 


\section{Introduction}

The case for an assessment of poverty considering multiple deprivations has been well argued for a long time. ${ }^{1}$ While there is a broad consensus about the multidimensionality of poverty, there is a debate as to whether the multiple indicators of deprivations should be brought together into a composite index or not. ${ }^{2}$ On the other hand, it seems that a composite measure of multiple deprivations is unavoidable when the purpose is to quantify the incidence of multiple deprivations within the same individuals. In practice, one of the approaches proposed to measure multidimensional poverty with a composite index is the

${ }^{1}$ See for instance, Sen (2001, chapter 4), and Sen (2009, chapter 12).

${ }^{2}$ Ravallion (2010), among others, discusses the pros and cons of each option. 
counting approach, which is based on counting the number of dimensions in which people are deprived. ${ }^{3}$ The approach has gained recent popularity with the Alkire-Foster (AF) family of poverty indices (Alkire and Foster, 2010). These indices identify the multidimensionally poor by counting the number of dimensions in which they are deprived. First, deprivation in any particular dimension is determined by comparing the achievement in that dimension against the respective dimension-specific poverty line. This is done for all dimensions/variables and then the (weighted) number of deprivations is compared against a multidimensionaldeprivation cut-off. ${ }^{4}$ By changing the cut-off from some minimum value up to the total number of dimensions, counting measures like the AF family can adopt identification criteria ranging from the union to the intersection approach. ${ }^{5}$ The intuitiveness and easy applicability of their identification and aggregation methods are reflected in the recent decision by the UNDP to estimate members of the AF family, including the adjusted headcount ratio, $M^{0}$, for the first time for 104 countries (See Alkire and Santos, 2010). This is part of an ongoing trend of the AF measures being applied in poverty measurement as well as in other fields unrelated to poverty measurement. ${ }^{6}$

An immediate concern with any composite index, including counting measures like the AF family, is that the orderings they produce, when comparing different groups, may not be robust to changes in the index's parameters. ${ }^{7}$ For instance, in the case of counting measures, changes in the dimension-specific poverty lines could reverse the rankings of different countries or provide contradictory results when ascertaining the direction of changes in poverty over time. With these concerns in mind, this paper derives the distributional conditions that ensure the robustness of ordinal comparisons to changes in the parameter values of counting measures characterized by relying on the weighted count of deprivations for the identification of the multidimensionally poor. Based on multivariate stochastic dominance techniques, the conditions are relevant for a broad range of counting measures including the AF family and most of the multidimensional poverty measures in the literature (e.g. Tsui (2002), Bourguignon and Chakravarty (2003), Chakravarty and D'Ambrosio (2006), Bossert et al. (2009)). The latter also rely on a counting approach, albeit some implicitly. The conditions are derived for poverty comparisons with ordinal variables (e.g. like the UNDP's MPI), because in such domain derivation is easiest. By contrast, applications to continuous variables (or continuous-discrete combinations) require more sophisticated techniques (e.g. generalized function theory), mainly due to the pervasiveness of discontinuities in the counting approach. These interesting problems are beyond the scope of this paper, and left for future research.

First, the paper provides full-robustness conditions for counting measures in bivariate settings. The conditions require making pairwise comparisons of cumulative and survival

\footnotetext{
${ }^{3}$ For a comparative discussion of approaches to measuring multidimensional poverty, see Atkinson (2003). For an alternative approach based on a multidimensional poverty line see Duclos et al. (2006, 2007).

${ }^{4}$ For instance, if considering 10 dimensions of wellbeing, a multidimensional deprivation cut-off of 5 means that a person is considered multidimensionally poor if the person is deprived in 5 or more of the 10 dimensions.

${ }^{5}$ According to the union approach, any person deprived in at least one dimension is considered multidimensionally poor. On the other extreme, the intersection approach demands considering as multidimensionally poor only people who are deprived in every dimension.

${ }^{6}$ For instance, Batana (2008), Santos and Ura (2008), Alkire and Seth (2008), Battiston et al. (2009), Foster et al. (2009), Azevedo and Robles (2009), Singh (2009), Trafton (2009) and Roche (2009).

${ }^{7}$ For a recent articulation of this concern see, for instance, Ravallion (2010).
} 
distributions. However, even though bivariate applications have been popular in poverty and wellbeing analysis (e.g. Atkinson and Bourguignon (1982), Duclos et al. (2006, 2007)), recent empirical applications of counting measures, chiefly (but not exclusively) the AlkireFoster family, consider more than two variables. The paper shows that, with three or more variables, robustness conditions based on multivariate generalizations of the work by Atkinson and Bourguignon (1982) are not applicable to counting measures, in general. However, for narrower classes of counting measures, robustness conditions can be derived. The restrictions characterizing the subfamily of counting measures for which multivariate dominance conditions are applicable, include focusing on extreme identification approaches: intersection and union.

An empirical application using the EU-SILC dataset illustrates the use of the conditions for ordinal variables. Measuring multidimensional poverty in terms of educational achievement, self-reported health, and ability to keep the house warm, it turns out that Austria, Finland, Sweden, Norway and the UK dominate three or more European countries whereas Italy, Latvia, Poland, Portugal and Spain are dominated by three or more countries.

The next section introduces the notation and the family of counting measures for which robustness conditions are sought. The subsequent section provides the robustness condition, in the form of a first-order dominance condition, for the whole family of counting measures in applications with bivariate distributions. Then the problem of applying traditional dominance conditions to three or more variables is discussed in the fourth section. The subsequent section shows the conditions that can be derived, when three or more variables are considered, by narrowing the class of counting measures to more explicit functions. This is followed by the empirical application and some concluding remarks.

\section{A general family of counting measures}

Consider a matrix $X$, whose $N$ rows have information on the attainments of $N$ individuals. Each column, therefore, hosts the distribution of each attainment across the population. The number of columns/variables is $D$. A typical attainment element of the matrix is: $x_{n d}\left(\in \mathbb{N}_{+}\right)$, that is, the attainment of individual $n$ in dimension/variable $d$. The poverty lines, specific to each variable, are denoted by $z_{d} ;{ }^{8}$ and a person is deemed poor in variable $d$ if: $x_{n d}<z_{d}$. The count of deprivations is computed weighting each deprivation with weights, $w_{d}$, such that: $w_{d} \in \mathbb{R}_{+} \wedge \sum_{d}^{D} w_{d}=D .^{9}$ Hence the weighted number of deprivations suffered by individual $n$ is: $c_{n} \equiv \sum_{d=1}^{D} w_{d} I\left(z_{d}>x_{n d}\right) \cdot{ }^{10}$ This paper considers the following family of counting measures that rely on the weighted deprivation count, $c_{n}$, for the identification of the multidimensionally poor:

$$
P \equiv \frac{1}{N} \sum_{n=1}^{N} p\left(c_{n} ; x_{n 1}, \ldots, x_{n D}, Z\right)=\frac{1}{N} \sum_{n=1}^{N} \varphi\left(c_{n}\right) g\left(\left[\frac{x_{n 1}}{z_{1}}\right]_{+}, \ldots,\left[\frac{x_{n D}}{z_{D}}\right]_{+}\right)
$$

\footnotetext{
${ }^{8}$ From a vector of poverty lines, $Z:\left(z_{1}, \ldots, z_{d}, \ldots, z_{D}\right)$.

${ }^{9}$ From a vector of weights, $W:\left(w_{1}, \ldots, w_{d}, \ldots, w_{D}\right)$.

${ }^{10} I()$ is an indicator function equal to 1 if the expression in parenthesis is true. Otherwise it takes the value of 0 .
} 
where $p$ is the individual poverty function and $\varphi:[0, D] \rightarrow\{0,1\}, \varphi^{\prime} \leq 0$, is the poverty identification function. $\varphi=1$ if (and only if) the individual is deemed multidimensionally poor. $\left[\frac{x_{n d}}{z_{d}}\right]_{+} \equiv \min \left\{1, \frac{x_{n d}}{z_{d}}\right\}$, and $g:[0,1]^{D} \rightarrow \mathbb{R}_{+}$is the part of $p$ that responds to the gaps between the attainments in each variable and their respective poverty lines, such that: $g^{\prime} \leq 0 ; g(1, \ldots, 1)=0$ and $\max g=1$. The choice of a social poverty function in the form of a social average of individual poverty functions, i.e. $P \equiv \frac{1}{N} \sum_{n=1}^{N} p$, is required for the purpose of applying the ordinal-variable equivalents of the multivariate extensions of Atkinson and Bourguignon (1982) to the derivation of robustness conditions, since their dominance conditions apply to social welfare functions that are "additively separable and symmetric with respect to individuals"(Atkinson and Bourguignon, 1982, p. 190). ${ }^{11}$

Lasso de la Vega (2010) shows that $\varphi\left(c_{n}\right)=I\left(c_{n} \geq k\right)$, where $k \in[0, D]$, is the only "non trivial dichotomized identification function" (p. 6) that fulfills a property of poverty consistency. A poverty identification function is poverty consistent if, when identifying a person with a deprivation count equal to $m$ as poor, it also considers as poor anybody whose count is at least as high as $m$. Considering the desirability of this property, this paper focuses on counting measures whose identification function has the form: $\varphi\left(c_{n} ; k\right)=I\left(c_{n} \geq k\right) \cdot{ }^{12}$ As for the form $g\left(\frac{x_{n 1}}{z_{1}}, \ldots, \frac{x_{n D}}{z_{D}}\right)$, all the multidimensional poverty functions in the literature depend on the ratios $\frac{x_{n d}}{z_{d}}$, as opposed to alternatives. It ensures their fulfilment of the desirable property of scale invariance.

Measures belonging to the class defined by (1), with $\varphi\left(c_{n}\right)=I\left(c_{n} \geq k\right)$, include the whole AF family, plus most of the multidimensional poverty indices proposed in the literature. While the AF family explicitly adopts a dichotomized identification function, i.e. $I\left(c_{n} \geq k\right)$, other multidimensional poverty indices can also be expressed as functions of such identification functions. Table (1) provides a list of the multidimensional poverty measures that work with ordinal variables and belong to the general class described in this section. ${ }^{13}$

To summarize, the measures of the ordinal-variable class (1) include all the measures in the multidimensional poverty literature that are applicable to ordinal variables . They are axiomatically characterized by the fulfillment of the following properties: additive decomposability, scale invariance, population replication invariance, weak monotonicity, dimensional monotonicity, poverty focus, deprivation focus, symmetry, normalization, weak rearrangement and poverty consistency. Except for the last one, all these properties are described by Alkire and Foster (2010). The last property has been formally studied by Lasso de la Vega (2010) .

\footnotetext{
${ }^{11}$ Their conditions are also applicable to poverty functions of the form: $R=h(P)$, where $h^{\prime}>0$. This is true because, for countries $A$ and $B, R^{A}>R^{B} \leftrightarrow P^{A}>P^{B}$. Some of the multidimensional poverty functions in the literature are of the $R$ form (e.g. Chakravarty and D'Ambrosio (2006), Bossert et al. (2009)).

${ }^{12}$ This two-stage, poverty identification function was introduced by Alkire and Foster (2010). The first stage involves the identification of deprivations in each variable/dimension. Then the second stage requires computing the weighted count of deprivations, $c_{n}$, and then comparing it against a cut-off value $k$. If $c_{n} \geq k$ the the person $n$ is identified as multidimensionally poor.

${ }^{13}$ In the table, only $g()$ denotes an implicit function that depends on the arguments within the parenthesis.
} 


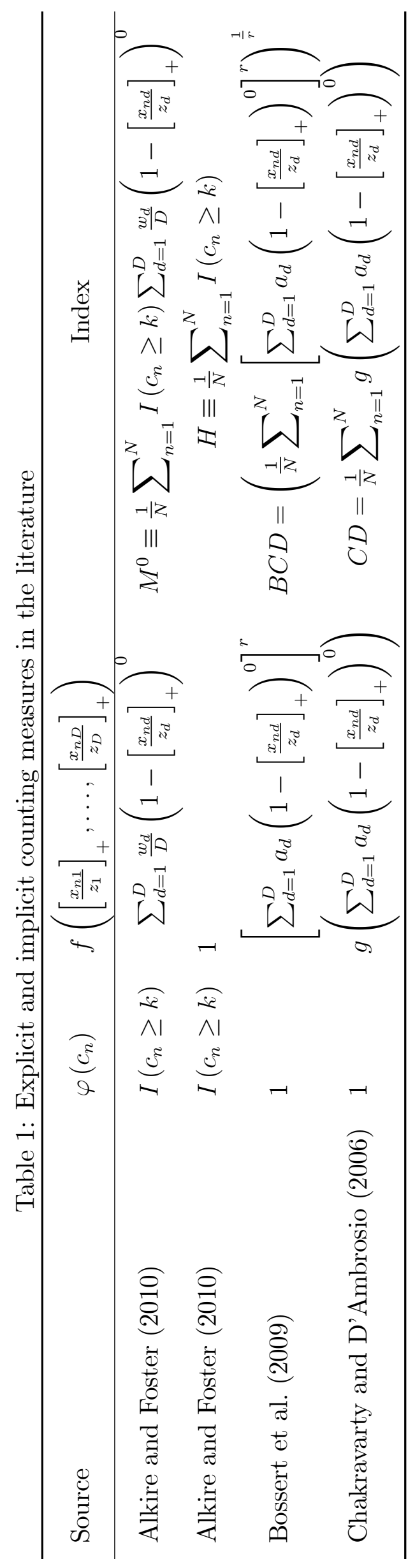




\section{The bivariate case}

\section{An intuitive derivation}

Consider countries $A$ and $B$. Under which distributional circumstances $P^{A}>P^{B}$ for any choice of weights $(W)$, poverty lines $(Z)$ and multidimensional cut-offs $(k)$ ? This is a problem of finding suitable first-order stochastic dominance conditions that can be applied to members of the class (1). Due to the arguments mentioned in the previous section, it is possible, in principle, to follow Atkinson and Bourguignon (1982) and integrate, or sum, by parts (1) and derive the required full-robustness conditions that ensure $P^{A}>P^{B}$. For such purpose (1) must be expressed either as a function of a density function (in the case of continuous variables) or as a function of a probability distribution function (in the case of ordinal variables). Since in this paper, the focus is on ordinal variables, the following version of (1) is considered:

$$
\begin{aligned}
P & \equiv \sum_{i=1}^{d_{1}} \ldots \sum_{m=1}^{d_{D}} \operatorname{Pr}\left[x_{1}=i, \ldots, x_{D}=m\right] p(i, \ldots, m ; W, Z, k) \\
& =\sum_{i=1}^{d_{1}} \ldots \sum_{m=1}^{d_{D}} \operatorname{Pr}\left[x_{1}=i, \ldots, x_{D}=m\right] \varphi(c ; k) g\left(\left[\frac{i}{z_{1}}\right]_{+}, \ldots,\left[\frac{m}{z_{D}}\right]_{+}\right),
\end{aligned}
$$

where the subscript $n$ is no longer necessary. While $P$ is the social poverty function analogue to a social welfare function, $p$ is the individual poverty analogue of an individual welfare function. Hence a first-order stochastic dominance condition for the class could be derived by summing by parts. But since these dominance results are known, ${ }^{14}$ in this section the condition is derived intuitively for the case of two variables, relying on the following two results:

1. The difference of the individual welfare function, $p\left(x_{1}, x_{2} ; W, Z, k\right)$, with respect to $x_{1}$ is non-positive:

$$
p_{1}=g \varphi_{1}+g_{1} \varphi \leq 0
$$

where $p_{1}(i, \ldots, m ; W, Z, k) \equiv p(i, \ldots, m ; W, Z, k)-p(i-1, \ldots, m ; W, Z, k)$; and similar definitions apply, e.g. for $g_{1}$ and $\varphi_{1}$. Now $p_{1} \leq 0$ because, by definition, $g_{1} \leq 0$, and $\varphi_{1} \leq 0$ since:

$$
\varphi_{1}(i)=-I\left(w_{2} I\left(x_{2} \leq z_{2}\right)<k\right) I\left(i-1<z_{1}<i\right)
$$

Expression (4) states that a change in $x_{1}$ changes the individual multidimensional poverty status if and only if being poor in $x_{2}$ is not sufficient to be deemed multidimensionally poor and the change in $x_{1}$ also changes the deprivation status with respect to $x_{1}$. Otherwise no change in the indicator function is produced. In either case, the derivative is non-positive $\left(\uparrow x_{d} \rightarrow \downarrow I\right.$, as it were).

\footnotetext{
${ }^{14}$ Atkinson and Bourguignon (1982) define the social welfare functions as integrals of individual welfare functions, and integrate them by parts in order to derive the dominance conditions. Yalonetzky (2013) shows that analogue results can be derived for social welfare functions based on ordinal variables, and expressed as sums, instead of integrals. In that case summation by parts is required.
} 
2. The cross-partial difference, $p_{12}\left(c ; i, j, \ldots, x_{D}, Z\right)$, can be either positive, negative, or equal to zero:

$$
p_{12}=g \varphi_{12}+g_{2} \varphi_{1}+g_{1} \varphi_{2}+g_{12} \varphi
$$

Note in (5) that the second and third right-hand side elements are non-negative but the fourth element could take any sign (because of the undefined sign of $g_{12}$ ), and also the first element could take any sign because, with two variables:

$$
\varphi_{12}=I\left(w_{1}<k \wedge w_{2}<k\right)-I\left(w_{1}>k \wedge w_{2}>k\right)
$$

Expression (6) indicates the circumstances under which the difference $\varphi_{1}$ may change from 0 to -1 , and vice versa, when $x_{2}$ changes. It also states the circumstances under which the difference $\varphi_{2}$ may change from 0 to -1 , and vice versa, when $x_{1}$ changes. The different signs that this cross-partial difference can take reflect the different ways in which the two variables may affect each other's effect on multidimensional poverty. For instance, when poverty identification follows the intersection approach $\left(w_{1}<k \wedge w_{2}<k\right)$, then the impact of an increase in $x_{1}$ is eliminated by a previous increase in $x_{2}$ if the person was poor to begin with (expression (4) changes value from -1 to 0 ). This is a case of ALEP substitution. ${ }^{15}$ By contrast, when poverty identification follows the union approach $\left(w_{1}>k \wedge w_{2}>k\right)$, the impact of an increase in $x_{1}$ is enhanced by a previous increase in $x_{2}$ if the person was poor to begin with (expression (4) changes value from 0 to -1 ). The latter is an example of ALEP complementarity, in which the cross-partial difference (6) is negative.

Now, in the bivariate stochastic dominance literature there are four well-established firstorder conditions, all of which are relevant to counting measures. The four conditions for ordinal variables stem from the following equations: ${ }^{16}$

$$
\begin{aligned}
\Delta S= & -\sum_{i=1}^{d_{1}} U_{1}\left(i, d_{2}\right) \Delta F_{1}(i-1)-\sum_{j=1}^{d_{2}} U_{2}\left(d_{1}, j\right) \Delta F_{1}(j-1) \\
& +\sum_{i=1}^{d_{1}} \sum_{i=j}^{d_{2}} U_{12}(i, j) \Delta F_{12}(i-1, j-1)
\end{aligned}
$$

and:

$$
\begin{aligned}
\Delta S= & \sum_{i=1}^{d_{1}} U_{1}(i, 1) \Delta \overline{F_{1}}(i)+\sum_{i=1}^{d_{2}} U_{2}(1, j) \Delta \overline{F_{2}}(j) \\
& +\sum_{i=1}^{d_{1}} \sum_{i=1}^{d_{2}} U_{12}(i, j) \Delta \overline{F_{12}}(i, j)
\end{aligned}
$$

where $U_{d}$ is the difference of an individual welfare function $U$ with respect to variable $x_{d}$; $\Delta S \equiv S^{A}-S^{B}$, and $S$ is a social welfare function, and $\overline{F_{i}}, \overline{F_{i j}}$, etc. are survival functions.

\footnotetext{
${ }^{15}$ For a definition of ALEP substitution and complementarity see Kannai (1980).

${ }^{16}$ See Yalonetzky (2013).
} 
A condition associated with $\Delta F_{12}(i, j), \Delta F_{1}, \Delta F_{2} \leq 0 \forall i, j \in\left[1, d_{1}\right] \times\left[1, d_{2}\right]$, requires the cross-partial difference to be negative, i.e. the two variables must be ALEP substitutes. An alternative condition, associated with $\Delta \overline{F_{12}}(i, j), \Delta \bar{F}_{1}, \Delta \bar{F}_{2} \geq 0 \forall i, j \in\left[1, d_{1}\right] \times\left[1, d_{2}\right]$, requires the cross-partial difference to be positive, i.e. the variables must be ALEP complements. A third condition stems from merging the first two distributional conditions. If these are met simultaneously, then the comparison, $\Delta S$, is robust for all individual welfare functions $U$, characterized by weak increasing monotonicity with respect to each variable, that is $U_{1}(i,),. U_{2}(j,.) \geq 0 \forall i, j \in\left[1, d_{1}\right] \times\left[1, d_{2}\right]$. The condition is stringent, but once fulfilled it guarantees robustness for all increasingly monotonic functions irrespective of the sign of their cross-partial difference.

The first difference of $p$ is non-positive. Yet a society is better-off than another one when its value for $P$ is the lowest. Therefore the distributional conditions related to (7) and (8) are also relevant for $P$. Since the cross-partial differences of $p$ can take any sign, then only the third bivariate condition applies. The dominance condition then becomes:

$$
\begin{aligned}
P^{A} & \leq P^{B}, \forall k \in[0,2] \forall w_{d} \in \mathbb{R}_{+} \wedge \sum_{d=1}^{2} w_{d}=2 ; \forall Z \leftrightarrow \\
\Delta F_{12}(i, j), \Delta F_{1}, \Delta F_{2} & \leq 0 \forall i, j \in\left[1, d_{1}\right] \times\left[1, d_{2}\right] \\
\wedge \Delta \bar{F}_{12}\left(x_{1}, x_{2}\right), \Delta \bar{F}_{1}, \Delta \bar{F}_{2} & \geq 0 \forall i, j \in\left[1, d_{1}\right] \times\left[1, d_{2}\right]
\end{aligned}
$$

According to condition (9), multidimensional poverty in society $A$ is never higher than in $B$, as measured by any member of the counting family defined by (1), if and only if the joint cumulative probability function of $A$ is never above that of $B$, and the joint survival probability function of $A$ is never below that of $B$, for all choices of specific poverty lines $(Z)$, weights $(W)$, multidimensional poverty cut-offs $(k)$ and $g$ functions.

\section{The case of three or more variables}

In this section I show why the above results cannot be extended to the general case of counting measures with three or more variables. In the next sections I show why and how the approach is applicable to counting measures and any multivariate distribution as long as extreme identification approaches are considered (i.e. union or intersection) and more structure is imposed on $g$.

The reason why the results are not generally applicable to cases of three, or more, variables is that with more than two variables, the multivariate versions of (7) and (8), for first-order dominance conditions, require checking the signs of all cross-partial differences involving all combinations of variables (i.e. including three and more variables). Existing multivariate conditions that work on cumulative and survival functions can handle any sign of cross-partial differences involving even numbers of variables. However, for odd numbers of variables (e.g. 1, 3, 5, etc.) the conditions only apply to non-negative cross-partial differences (or non-positive, in the case of poverty functions). This is clear by examining the multivariate versions of (7) and (8): 


$$
\begin{aligned}
\Delta S= & -\sum_{a=1}^{D} \sum_{i=1}^{d_{a}} U_{a}\left(i, \ldots, d_{b \neq a}\right) \Delta F_{a}(i-1) \\
& +\sum_{a=1}^{D-1} \sum_{b=a+1}^{D} \sum_{i=1}^{d_{a}} \sum_{j=1}^{d_{b}} U_{a b}\left(i, j, \ldots d_{c \neq a, b}\right) \Delta F_{a b}(i-1, j-1) \\
& -\sum_{a=1}^{D-2} \sum_{b=a+1}^{D-1} \sum_{c=b+1}^{D} \sum_{i=1}^{d_{a}} \sum_{j=1}^{d_{b}} \sum_{l=1}^{d_{c}} U_{a b c}\left(i, j, l, \ldots, d_{u \neq a, b, c}\right) \Delta F_{a b c}(i-1, j-1, l-1) \cdots \\
& +(-1)^{D} \sum_{i=1}^{d_{1}} \ldots \sum_{l=1}^{d_{D}} U_{1 \ldots D} \Delta F(i, \ldots, l)
\end{aligned}
$$

and:

$$
\begin{aligned}
\Delta S= & \sum_{a=1}^{D} \sum_{i=1}^{d_{a}} U_{a}(i, \ldots, 1) \Delta \overline{F_{a}}(i) \\
& +\sum_{a=1}^{D-1} \sum_{b=a+1}^{D} \sum_{i=1}^{d_{a}} \sum_{j=1}^{d_{b}} U_{a b}(i, j, \ldots 1) \Delta \overline{F_{a b}}(i, j) \\
& +\sum_{a=1}^{D-2} \sum_{b=a+1}^{D-1} \sum_{c=b+1}^{D} \sum_{i=1}^{d_{a}} \sum_{j=1}^{d_{b}} \sum_{l=1}^{d_{c}} U_{a b c}(i, j, l, \ldots, 1) \Delta \overline{F_{a b c}}(i, j, l) \ldots \\
& +\sum_{i=1}^{d_{1}} \ldots \sum_{l=1}^{d_{D}} U_{1 \ldots D} \Delta \bar{F}(i, \ldots, l)
\end{aligned}
$$

where the notation is the same as in $(7)$ and $(8) .{ }^{17}$

A condition associated with $\Delta F, \ldots, \Delta F_{a b c}, \ldots, \Delta F_{a} \leq 0 \forall i, \ldots, l \in\left[1, d_{1}\right] \times \ldots\left[1, d_{D}\right]$, requires that the cross-partial differences alternate signs starting with $U_{a}\left(i, \ldots, d_{b \neq a}\right) \geq 0$, followed by $U_{a b}\left(i, j, \ldots, d_{c \neq a, b}\right) \leq 0$, and so on until $U_{1 \ldots D} \leq 0$ if $D$ is an even number, or $U_{1 \ldots D} \geq 0$ otherwise. An alternative condition, associated with $\Delta \bar{F}, \ldots, \Delta \overline{F_{a b c}}, \ldots \Delta \bar{F}_{a} \geq$ $0 \forall i, \ldots, l \in\left[1, d_{1}\right] \times \ldots\left[1, d_{D}\right]$, requires that all cross-partial differences be non-negative. A third condition stems from the merger of the first two distributional conditions. If the two first conditions are met, then the comparison, $\Delta S$, is robust for all individual welfare functions $U$, characterized by weak increasing monotonicity with respect to each variable, $\left(U_{a}(i, \ldots) \geq 0 \forall a\right)$ and by positively (or zero) signed cross-partial derivatives for odd numbers of variables. Hence, for the conditions stemming from (10) and (11) to be suitable for the derivation of a multivariate version of (9), it is necessary that the odd cross-partial differences of $p$ be non-positive (because it is a poverty function). By examining the simplest

\footnotetext{
${ }^{17}$ The result (10) has been shown by Crawford (2005), although it was alluded to by Hadar and Russell (1974). The result (11) is a simple multidimensional extension of the three dimensional derivation by Anderson (2008).
} 
cross-partial difference it is easy to realize that the conditions are not suitable. First, the multivariate equivalent of (3), i.e. the partial difference is non-positive since $g_{1} \leq 0$ and:

$$
\varphi_{1}(i)=-I\left(c_{n}-w_{1}<k\right) I\left(i-1<z_{1}<i\right)
$$

The difference (12) does not pose any problem. By contrast, the multivariate equivalent of (5) can also take any sign. Its expression is more complicated. ${ }^{18}$ With some further manipulation one can show that $\varphi_{123}(i, j, l)$ can also take any sign (not just non-positive) and hence $p_{123}$ can take any sign. Therefore neither (10) nor (11) provide suitable dominance conditions for the class of poverty functions based on (1) when more than two variables are considered, in general. However, as the next section shows, by restricting the identification criteria to the extreme approaches and imposing more structure on $g$, the potential signs of the cross-partial differences of $p$ can be narrowed down so that first-order dominance conditions become suitable for certain subsets of members of (1). As illustrated below, some of the counting measures in the literature belong to these subfamilies.

\section{Conditions for three or more variables}

Since one of the reasons why the multivariate equivalent of (5) can take any sign is that the cross-partial differences of $\varphi(c ; k)$ can take any sign, then a natural starting point for finding subfamilies of $P$ for which dominance conditions are applicable, is to narrow down the poverty identification criteria, i.e. $\varphi(c ; k)$. The first subsection of this section derives the subfamilies of $P$ for which full-robustness conditions are available from first-order multivariate dominance conditions, when the identification of the poor is restricted to the union approach (as several indices in the literature do). The second subsection focuses on the intersection approach. Any alternative, intermediate identification approach is characterized by cross-partial differences of $\varphi(c ; k)$ that can take any sign. Hence the first-order multivariate extensions of Atkinson and Bourguignon (1982) only apply to the extreme identification approaches when three, or more, variables are considered.

\section{The case of the union approach}

In the union approach, $\varphi(c ; k)=I\left(c_{n} \geq \min \left\{w_{d}\right\}\right)=I\left(\exists s \mid x_{n s}<z_{s}\right)$. Hence:

$$
\begin{aligned}
P\left(k=\min \left\{w_{d}\right\}\right) & =\sum_{i=1}^{d_{1}} \ldots \sum_{m=1}^{d_{D}} \operatorname{Pr}\left[x_{1}=i, \ldots, x_{D}=m\right] \varphi\left(c ; \min \left\{w_{d}\right\}\right) g\left(\left[\frac{x_{1}}{z_{1}}\right]_{+}, \ldots,\left[\frac{x_{D}}{z_{D}}\right]_{+}\right) \\
& =\sum_{i=1}^{d_{1}} \ldots \sum_{m=1}^{d_{D}} \operatorname{Pr}\left[x_{1}=i, \ldots, x_{D}=m\right] g\left(\left[\frac{x_{1}}{z_{1}}\right]_{+}, \ldots,\left[\frac{x_{D}}{z_{D}}\right]_{+}\right)=P(k=0)
\end{aligned}
$$

\footnotetext{
${ }^{18}$ Because it involves $\varphi_{12}(i, j)$, which depends not just on the two differentiation variables. The formula appears in the Appendix 1.
} 
Note that, in the second line of $(13), \varphi\left(c ; \min \left\{w_{d}\right\}\right)$ dropped out. The reason is that, under the union approach, $g=0$ for all the non-poor, because in their case: $x_{s} \geq z_{s} \forall s \in$ $[1, D]$.Therefore, due to the properties fulfilled by $g$, the computation of $P$ as if everybody were identified as poor $(\varphi(c ; 0))$ always yields the same value as in the case of the union approach. The implication, then, is that the first-order dominance conditions for $P(k=0)$ are the correct robustness conditions for $P\left(k=\min \left\{w_{d}\right\}\right)$.

Now the first-order dominance conditions for $P(k=0)$ only depend on the cross-partial differences of $g$. That is, the sign of the cross-partial differences of $p$ depend exclusively on the signs of the cross-partial differences of $g$; because, when $k=0: p=g$. Without further structure, the signs of the cross-partial differences of $g$ are ambiguous. Hence multivariate dominance conditions are not applicable for three, or more, variables even in the union approach. Consequently the search for subfamilies of $P$ for which dominance conditions are applicable, under the union approach, must be restricted to functions $g$ whose cross-partial differences take different non-ambiguous signs. By an induction argument (i.e. using the patterns stemming from the cross-partial differences of $g$ ), the following dominance condition, and corresponding subfamily of $P$ measures, can be derived:

$$
\begin{aligned}
P^{A} & \leq P^{B}, \forall w_{d} \in \mathbb{R}_{+} \wedge \sum_{d=1}^{D} w_{d}=D ; \forall Z ; k=\min \left\{w_{d}\right\} ; \leftrightarrow \\
\Delta F(i, \ldots, l), \Delta F_{a b c}(i, j, l), \ldots \Delta F_{a} & \leq 0 \forall i, \ldots, l \in\left[1, d_{1}\right] \times \ldots\left[1, d_{D}\right] \\
\wedge g_{a}, g_{a b c}, \ldots & \leq 0 ; g_{a b}, g_{a b c d}, \ldots \geq 0
\end{aligned}
$$

According to condition (14), multidimensional poverty in society $A$ is never higher than in $B$, as measured by members of a subclass of the counting family (1) characterized by the union approach, non-positive, odd-numbered cross-partial differences of $g$ and non-negative, even-numbered cross partial differences of $g$, if and only if the joint cumulative probability function of $A$ is never above that of $B$, for all choices of specific poverty lines $(Z)$ and weights $(W)$.

An example of $g$ for which condition (14) applies is: $g=h\left(\sum_{d=1}^{D} a_{d} K\left(\left[\frac{x_{n d}}{z_{d}}\right]_{+}\right)\right)$, where $h: \mathbb{R}_{+} \rightarrow \mathbb{R}_{+}, K:[0,1] \rightarrow \mathbb{R}_{+}, a_{d} \in \mathbb{R}_{+} \wedge \sum_{d=1}^{D} a_{d}=D, K^{\prime} \leq 0$ and the cross-partial differences of $h$ are non-negative if they are odd-numbered and non-positive if they are evennumbered. Such an example can be provided by several generalized means functions, e.g. expression (18) in Bourguignon and Chakravarty (2003).

Two additional conditions for the union approach, and three or more variables, can be derived, departing from (14). By relaxing the requirements on the cross-partial differences of $g$ a more stringent distributional condition is available for a broader subfamily of $P$ : 


$$
\begin{aligned}
P^{A} & \leq P^{B}, \forall w_{d} \in \mathbb{R}_{+} \wedge \sum_{d=1}^{D} w_{d}=D ; \forall Z ; k=\min \left\{w_{d}\right\} ; \leftrightarrow \\
\Delta F(i, \ldots, l), \Delta F_{a b c}(i, j, l), \ldots \Delta F_{a} & \leq 0 \forall i, \ldots, l \in\left[1, d_{1}\right] \times \ldots\left[1, d_{D}\right] \\
\wedge \Delta \bar{F}(i, \ldots, l), \Delta \overline{F_{a b c}}(i, j, l), \ldots \Delta \overline{F_{a}} & \geq 0 \forall i, \ldots, l \in\left[1, d_{1}\right] \times \ldots\left[1, d_{D}\right] \\
\wedge g_{a}, g_{a b c}, \ldots & \leq 0
\end{aligned}
$$

According to condition (15), multidimensional poverty in society $A$ is never higher than in $B$, as measured by members of a subclass of the counting family (1) characterized by the union approach, and non-positive, odd-numbered cross-partial differences of $g$, if and only if the joint cumulative probability function of $A$ is never above that of $B$ and the joint survival probability function of $A$ is never below that of $B$, for all choices of specific poverty lines $(Z)$ and weights $(W)$.

Finally, the second additional condition stems from setting all cross-partial differences of $g$ equal to zero. In that case, the full-robustness condition is:

$$
\begin{aligned}
P^{A} & \leq P^{B}, \forall w_{d} \in \mathbb{R}_{+} \wedge \sum_{d=1}^{D} w_{d}=D ; \forall Z ; k=\min \left\{w_{d}\right\} ; \leftrightarrow \\
\Delta F_{a} & \leq 0 \forall i, \ldots, l \in\left[1, d_{1}\right] \times \ldots\left[1, d_{D}\right] \\
\wedge g_{a b}, g_{a b c}, g_{a b c d} \ldots & =0
\end{aligned}
$$

Robustness condition (16) applies to members of $P$ whose $g$ is additively separable into $D$ arguments that depend on each of the $\left[\frac{x_{n d}}{z_{d}}\right]_{+}$, respectively. ${ }^{19}$ A prominent example of these measures is the Alkire-Foster family. Another example is provided by $B C D$ when $\gamma=1$ (see Table 1, Bossert et al. (2009)). Hence, under the union approach, condition (16) is applicable to the Alkire-Foster family, among others. The condition is only sensitive to the marginal distributions of the variables. It requires testing for first-order dominance over each variable separately and declaring dominance only when one society dominates another one in each and every variable, independently.

\section{The case of the intersection approach}

In the case of the intersection approach: $\varphi\left(c_{n} ; D\right)=I\left(\forall s: x_{n s}<z_{s}\right)$. Then the cross-partial differences of $\varphi$ follow a pattern whereby the odd-numbered differences are always nonpositive and the even-numbered ones are non-negative, i.e. possible signs are alternated. For instance the first three cross-partial differencess are:

$$
\begin{gathered}
\varphi_{1}(k=D)=-I\left(c_{n / 1}=D-w_{1}\right) I\left(i-1<z_{1}<i\right) ; \\
\text { where } \quad c_{n / 1} \equiv \sum_{d \neq 1}^{D} w_{d} I\left(x_{n d}<z_{d}\right)
\end{gathered}
$$

\footnotetext{
${ }^{19} \mathrm{I}$ am using additive separability in the same sense as Gorman (1968).
} 


$$
\begin{gathered}
\varphi_{12}(k=D)=I\left(c_{n / 1,2}=D-w_{1}-w_{2}\right) I\left(i-1<z_{1}<i\right) I\left(j-1<z_{2}<j\right) ; \quad \text { (18) } \\
\text { where } \quad c_{n / 1,2} \equiv \sum_{d \neq 1,2}^{D} w_{d} I\left(x_{n d}<z_{d}\right) \\
\varphi_{123}(k=D)=-I\left(c_{n / 1,2,3}=D-w_{1}-w_{2}-w_{3}\right) I\left(i-1<z_{1}<i\right) I\left(j-1<z_{2}<j\right) I\left(l-1<z_{3}<(\text { (1) }) ;\right. \\
\text { where } \quad c_{n / 1,2,3} \equiv \sum_{d \neq 1,2,3}^{D} w_{d} I\left(x_{n d}<z_{d}\right)
\end{gathered}
$$

Identifying the subfamily of counting measures for which multivariate dominance conditions apply, under the intersection approach, requires checking the signs that the cross-partial differences of $p$ can take. Considering the potential signs of the cross-partial differences of $\varphi$ under the intersection approach (e.g. (18) and (19)), an inspection of the simplest crosspartial differences of $p$ (see Appendix 2), shows that the cross-partial differences of $g$ also need to alternate potential signs in the same way as $\varphi$, i.e. : $\varphi_{a} \leq 0, \varphi_{a b} \geq 0, \varphi_{a b c} \leq 0$, and so forth. Otherwise the potential sign of the cross-partial differences of $p$ becomes ambiguous and first-order dominance conditions do not qualify as full-robustness conditions. Therefore, when the cross-partial differences of $g$ behave in a manner mirroring those of $\varphi$, the following condition, and corresponding subfamily of $P$, can be derived:

$$
\begin{aligned}
P^{A} & \leq P^{B}, \forall w_{d} \in \mathbb{R}_{+} \wedge \sum_{d=1}^{D} w_{d}=D ; \forall Z ; k=D ; \leftrightarrow(20) \\
\Delta F(i, \ldots, l), \Delta F_{a b c}(i, j, l), \ldots \Delta F_{a} & \leq 0 \forall i, \ldots, l \in\left[1, d_{1}\right] \times \ldots\left[1, d_{D}\right] \\
\wedge g_{a}, g_{a b c}, \ldots & \leq 0 ; g_{a b}, g_{a b c d}, \ldots \geq 0
\end{aligned}
$$

According to (20), multidimensional poverty in society $A$ is never higher than in $B$, as measured by members of a subclass of the counting family (1) characterized by the intersection approach, non-positive, odd-numbered cross-partial derivatives of $g$ and non-negative, even-numbered cross partial derivatives of $g$, if and only if the joint cumulative probability function of $A$ is never above that of $B$, for all choices of specific poverty lines $(Z)$ and weights $(W)$. Because condition (20) is identical to condition (14), but applied to the intersection approach, the examples of $g$ that are suitable for the latter, e.g. several generalized means functions, are also relevant for the former. For instance, condition (20) is also applicable to the Alkire-Foster family under the intersection approach.

\section{Empirical application}

An illustration of the conditions deduced above is provided in this section using the EU-SILC dataset. For the 26 European countries ${ }^{20}$ in the sample, the following three indicators of

\footnotetext{
${ }^{20}$ Austria, Belgium, Croatia, the Czech Republic, Germany, Denmark, Estonia, Spain, Finland, France, Greece, Hungary, Ireland, Iceland, Italy, Lithuania, Luxembourg, the Netherlands, Norway, Poland, Portugal, Sweden, Slovenia, Slovakia, and the United Kingdom.
} 
functionings for adults at least 25 years old are considered:

- Education measured by the highest International Standard Classification of Education (ISCED) level attained according to the following ordinal categories: pre-primary education, primary education, lower secondary education, upper secondary education, post-secondary non-tertiary education, first and second stages of tertiary education. ${ }^{21}$

- Health, self-reported using the following categories: very bad, bad, fair, good, very good.

- Ability to keep the home adequately warm (a dummy variable). ${ }^{22}$

The sample sizes for each country are in Table 5 in the Appendix 3.

\section{Results}

Tables 2 and 3 show the dominance test results for dominance conditions based on joint cumulative distributions and joint survival distributions, respectively. The tests are the ones proposed by Yalonetzky (2013). In these tests two sets of hypotheses are tested. First, a null hypothesis of distributional homogeneity between $\mathrm{A}$ and $\mathrm{B}$ is tested against the alternative of strict dominance of A over B; second, a null hypothesis of homogeneity of A and B is rejected against the alternative hypothesis of curve-crossing. The tests rely on z-statistics and, for the first set, rejection is concluded when all statistics are of the same sign and statistically significant. For the second set, rejection is concluded when there are at least two statistically significant statistics of different sign. ${ }^{23}$ Note from the tables that, in most cases, there are statistically significant curve-crossings.

Meanwhile, with three or more variables, a multidimensional poverty comparison based on any measure belonging to (1), and on extreme poverty identification approaches, is fully robust if and only if the two dominance conditions are met simultaneously. Table 4 provides a summary of the pairs of countries for which both conditions are met, hence guaranteeing full robustness of multidimensional poverty comparisons for the functioning indicators considered. ${ }^{24}$ It turns out that Austria, Finland, Sweden, Norway and the UK dominate three or more European countries; whereas Italy, Latvia, Poland, Portugal and Spain are dominated by three or more countries. By contrast, most of the comparisons are not fully robust. Hence, for instance, whether Austria is poorer than Belgium or the other way around, depends on the choice of poverty measure and/or its specific parameter values.

\footnotetext{
${ }^{21}$ The first stage does not lead to an advance research qualification, whereas the second stage does. See EUROSTAT (2007).

${ }^{22}$ This question is about the ability to keep the house adequately warm, including the ability to pay bills for that, independently of whether the house needs to be heated. See EUROSTAT (2007).

${ }^{23}$ See Yalonetzky (2013) for more details.

${ }^{24}$ The result shown in Table 4 is the one from the other two tables that exhibits the highest test size.
} 


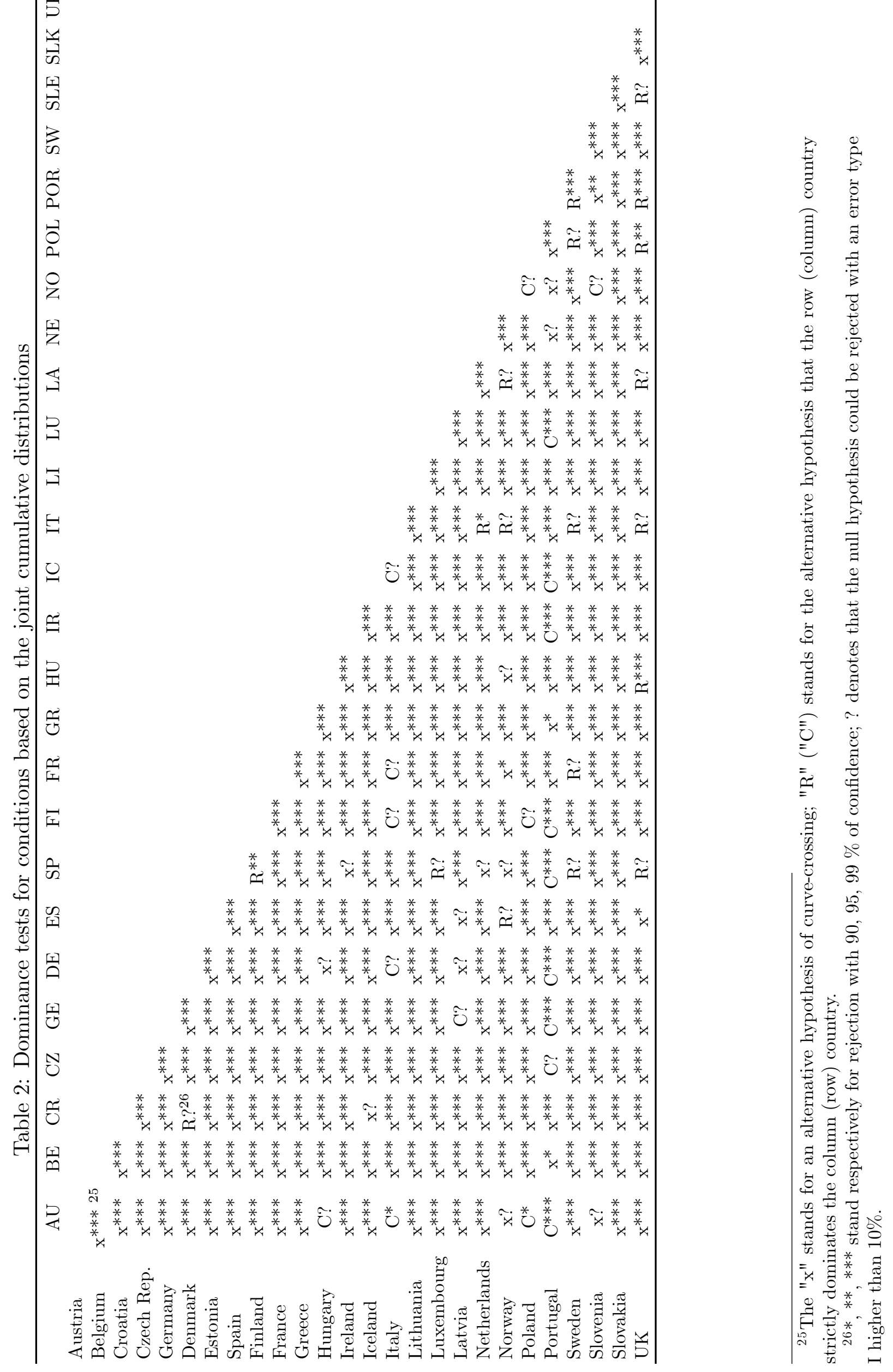




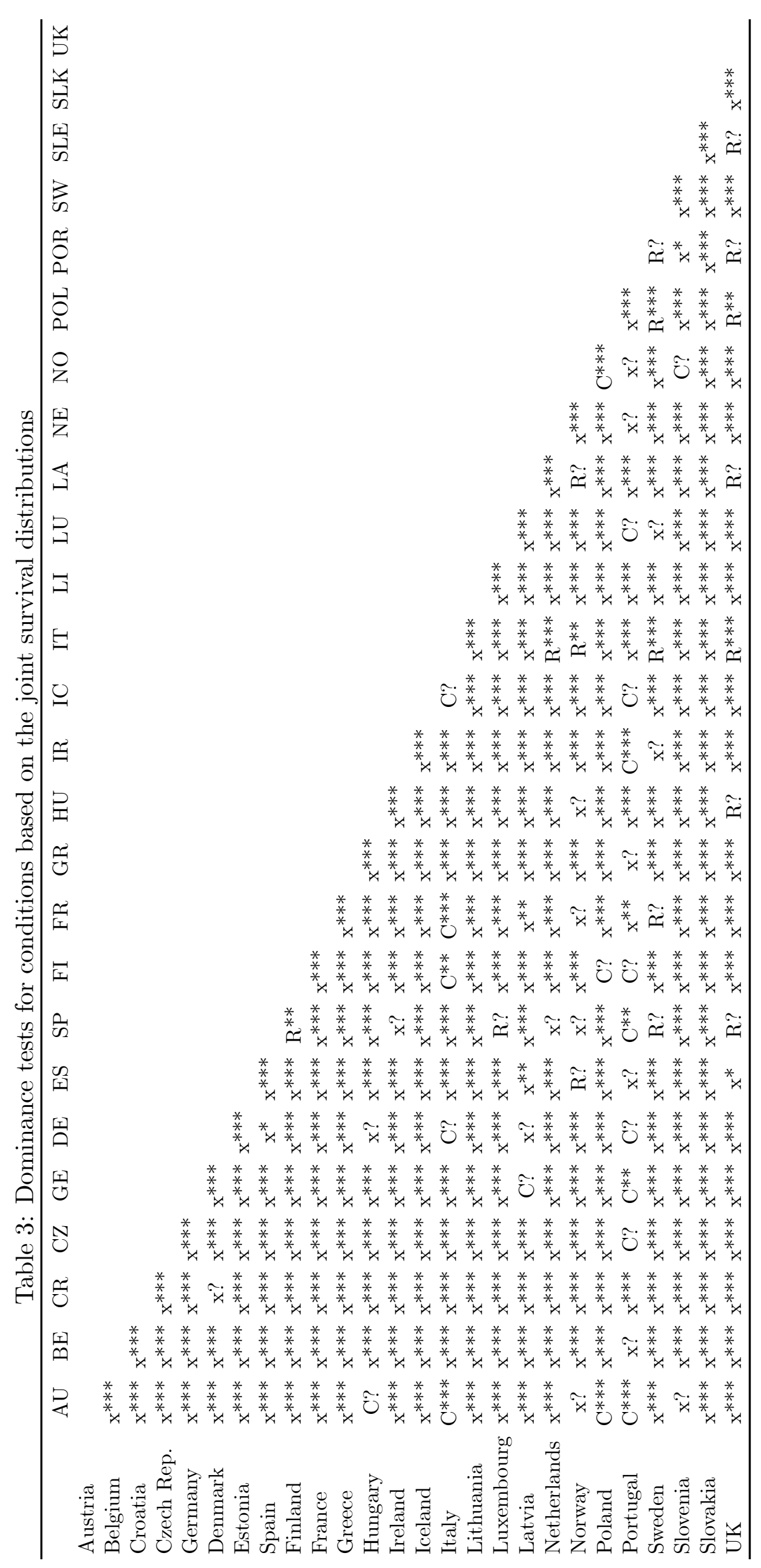




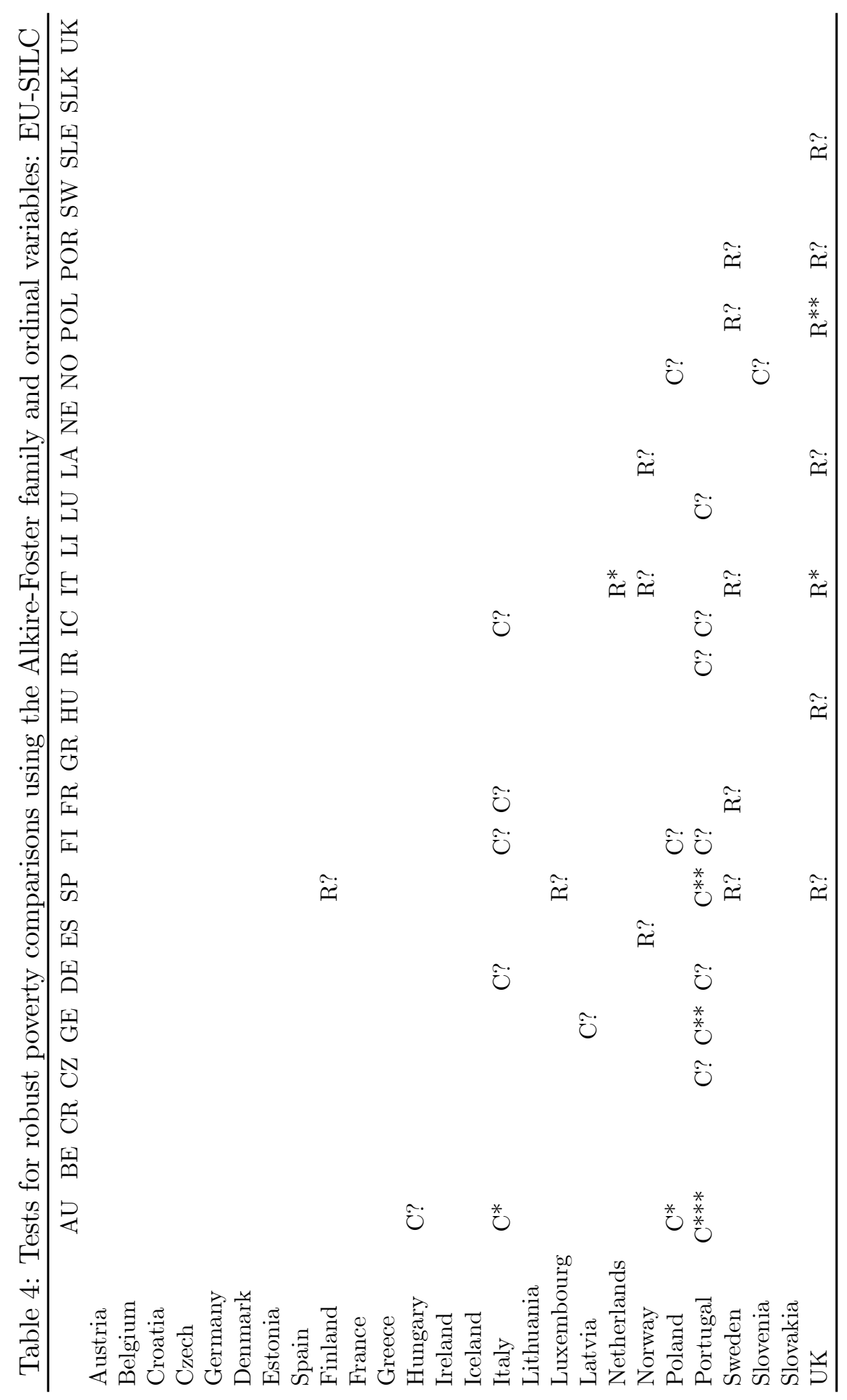




\section{Concluding remarks}

Previous work on the ordinal robustness of counting measures has either focused on bivariate applications of measures that are implicitly based on the counting approach (e.g. Bourguignon and Chakravarty (2003)) or focused on the robustness of comparisons to changes in the counting cut-off, $k$ (e.g. Lasso de la Vega (2010) and Alkire and Foster (2010)). This paper has sought to provide the distributional conditions that ensure the full robustness of an ordinal poverty comparison, based on ordinal variables and on a very broad family of counting measures that includes most measures of multidimensional poverty in the literature that depend, explicitly or implicitly, on the counting identification function characterized by Lasso de la Vega (2010). When fulfilled, these conditions ensure that poverty comparisons are robust to changes in the value of any of the parameters upon which the counting measures depend. The paper shows that, in bivariate cases, the conditions require comparing the joint cumulative and survival functions of two distributions. The conditions are stringent, but they provide the maximum degree of robustness in poverty comparisons using these measures.

In applications with three or more variables, the traditional dominance conditions used in this paper are not appropriate for poverty counting measures, except when extreme poverty identification approaches are considered. The reason is that the conditions do not cover all the possible signs that certain cross-partial differences may take; specifically, those of cross-partial differences of the individual poverty function with respect to an odd number of variables. For intermediate identification approaches, the odd cross-partial differences of poverty counting measures can take any sign. Hence traditional dominance conditions are not applicable.

However this paper shows that there are suitable dominance conditions for any number of variables, only for extreme poverty identification approaches. In the case of the union approach, the paper derives three first-order dominance conditions that are suitable as full robustness conditions. Each depends on different sets of signs that the cross-partial differences of $g$ can take. By contrast, in the case of the intersection approach, only one distributional condition can be derived. According to the latter, a country whose cumulative joint and marginal distributions first-order dominate will not exhibit higher poverty according to any of the counting measures belonging to $(1)$, with $\varphi\left(c_{n} ; k\right)=I\left(c_{n}=D\right)$.

What's left for poverty comparisons using counting measures, intermediate identification approaches and several ordinal variables? In these circumstances, traditional dominance conditions cannot ascertain robustness when fulfilled. However, by looking at (10) or (11), it is clear that when these conditions are not fulfilled, ordinal poverty comparisons with counting measures are not robust. This limitation in the applicability of multivariate first-order conditions to robustness of poverty comparisons is not a consequence of using counting measures. It's rather a reflection of the lack of resilience of traditional distributional conditions to deal with different signs of the odd-numbered cross partial differences (and derivatives). Hence the limited applicability of the conditions is bound to be a problem in other contexts of ordinal poverty and wellbeing comparisons. ${ }^{27}$ This state of affairs calls for future

\footnotetext{
${ }^{27}$ For instance, when using the new family of measures proposed by Rippin (2011). Her family is similar to (1), but $\varphi\left(c_{n}\right)$ takes a more general form and the union approach is adopted. The latter means that $\varphi\left(c_{n}\right)$
} 
research to explore alternative robustness criteria for ordinal poverty (and wellbeing) comparisons, beyond the traditional tools developed since the seminal contribution of Atkinson and Bourguignon (1982).

The empirical illustration relying on the EU-SILC dataset shows the existence of several robust comparisons of multidimensional poverty using educational achievement, self-reported health, and ability to keep the house warm. For instance, Austria, Finland, Sweden, Norway and the UK dominate three or more European countries; whereas Italy, Latvia, Poland, Portugal and Spain are dominated by three or more countries. However, as is often the case, a complete and fully robust ordering does not stem from these data. This means that several other comparisons, as well as the aim of generating any complete ordering, ultimately depend on the choices of multidimensional poverty measures and their parameters' values (e.g. specific poverty lines, weights and multidimensional cut-offs in the case of the AlkireFoster family of measures). It is possible that some other dominance relationship would emerge in this dataset should the test of the conditions be restricted, say, by ruling out values of the variables that appear not to be reasonable as poverty lines. However applying these restrictions when dealing with multiple variables is not trivial. Particularly one does not want to rule out testing conditions related to union approaches, i.e. in which low values of some variables are considered along with the whole range of other variables (since in these approaches, a person can be deemed poor even if he or she performs well in some dimensions

of wellbeing). Therefore this restricted-dominance approach has not been pursued in the paper.

This paper's focus has been on ordinal variables. Future research should also ascertain the validity of similar dominance conditions as robustness criteria for measures based on continuous variables. Hybrids depending on both continuous and discrete ordinal variables should also be considered. Given the pervasiveness of discontinuities in the functional forms of the continuous-equivalent of (1), the application of traditional stochastic dominance techniques is expected to be challenging in these contexts. Resorting to the theory of generalized functions; or, alternatively, smoothing the discontinuities around the poverty lines, may be the way to go.

\section{References}

Alkire, S. and J. Foster (2010). Counting and multidimensional poverty measurement. Journal of Public Economics (doi:10.1016). Forthcoming.

Alkire, S. and M. E. Santos (2010). Acute multidimensional poverty: A new index for developing countries. OPHI Working Paper 38.

Alkire, S. and S. Seth (2008). Measuring multidimensional poverty in india: a new proposal. OPHI Working Paper 15.

Anderson, G. (2008). The empirical assessment of multidimensional welfare, inequality and poverty: sample weighted multivariate generalizations of the Kolmogorov-Smirnov two sample test for stochastic dominance. Journal of Economic Inequality 6(1), 73-87.

ceases to be an identification function. 
Atkinson, A. (2003). Multidimensional deprivation: contrasting social welfare and counting approaches. Journal of Economic Inequality 1, 51-65.

Atkinson, A. and F. Bourguignon (1982). The comparison of multi-dimensioned distributions of economic status. Review of Economic Studies XLIX, 183-201.

Azevedo, V. and M. Robles (2009). Multdimensional targeting: identifying beneficiaries of poverty reduction programs. Manuscript.

Batana, Y. (2008). Multidimensional measurement of poverty in sub saharan africa. OPHI Working Paper 13.

Battiston, D., G. Cruces, L. F. Lopez-Calva, M. A. Lugo, and M. E. Santos (2009). Income and beyond: multidimensional poverty in six latin america. OPHI Working Paper 17.

Bossert, W., C. D'Ambrosio, and S. Chakravarty (2009). Measuring multidimensional poverty and material deprivation. ECINEQ Working Paper 2009-129.

Bourguignon, F. and S. Chakravarty (2003). The measurement of multidimensional poverty. Journal of Economic Inequality 1, 25-49.

Chakravarty, S. and C. D'Ambrosio (2006). The measurement of social exclusion. Review of Income and wealth 52(3), 377-98.

Crawford, I. (2005). A nonparametric test of stochastic dominance in multivariate distributions. manuscript.

Duclos, J.-Y., D. Sahn, and S. Younger (2006). Robust multidimensional poverty comparisons. The Economic Journal 116(514), 943-68.

Duclos, J.-Y., D. Sahn, and S. Younger (2007). Inequality and Poverty Re-examined, Chapter Robust multidimensional poverty comparisons with discrete indicators of well-being, pp. 185-208. Oxford University Press.

EUROSTAT (2007). Description of SILC user database variables: Cross-sectional and longitudinal. European Commission.

Foster, J., A. Horowitz, and F. Mendez (2009). An axiomatic approach to the measurement of corruption. OPHI Working Paper 29.

Gorman, W. (1968). Conditions for additive separability. Econometrica 36(3-4), 605-9.

Hadar, J. and W. Russell (1974). Essays on economic behaviour under uncertainty, Chapter Stochastic dominance in choice under uncertainty. North-Holland.

Kannai, Y. (1980). The alep definition of complementarity and least concave utility functions. Journal of Economic Theory 22(1), 115-7.

Lasso de la Vega, C. (2010). Counting poverty orderings and deprivation curves. In J. Bishop (Ed.), Research on Economic Inequality, Volume 18, Chapter 7, pp. 153-72. Emerald. 
Ravallion, M. (2010). Mashup indices of development. Policy Research Working Paper 5432.

Rippin, N. (2011). Multidimensional poverty measurement: the issue of inequality between dimensions.

Roche, J. M. (2009). Child poverty measurement: an assessment of methods and an application to bangladesh. Manuscript.

Santos, M. E. and K. Ura (2008). Multidimensional poverty in bhutan: estimates and policy implications. OPHI Working Paper 14.

Sen, A. (2001). Development as Freedom. Oxford University Press.

Sen, A. (2009). The Idea of Justice. Allen Lane.

Singh, S. (2009). Towards a multidimensional measure of governance. Manuscript.

Trafton, T. (2009). Multdimensional social outcome measurement. Manuscript.

Tsui, K.-Y. (2002). Multdimensional poverty indices. Social Choice and Welfare 19, 69-93.

Yalonetzky, G. (2013). Stochastic dominance with ordinal variables: Conditions and a test. Econometric Reviews DOI:10.1080/07474938.2012.690653.

\section{Appendix 1: Cross-partial difference of $\psi\left(c_{n} ; k\right)$}

$$
\begin{aligned}
\varphi_{12}(i, j)= & I\left(i-1<z_{1}<i\right) I\left(j-1<z_{2}<j\right) I\left(c_{n}-w_{1}-w_{2}<k\right)[ \\
& I\left(c_{n}-w_{1}<k\right) I\left(c_{n}-w_{2} \geq k\right)+I\left(c_{n}-w_{2}<k\right) I\left(c_{n}-w_{1} \geq k\right) \\
& \left.-I\left(c_{n}-w_{1}<k\right) I\left(c_{n}-w_{2}<k\right)\right]
\end{aligned}
$$

\section{Appendix 2: Basic cross-partial differences of $p$ in the intersection approach}

$$
\begin{aligned}
p_{12}= & g \varphi_{12}\left(c_{n} ; D\right)+g_{1} \varphi_{2}\left(c_{n} ; D\right)+g_{2} \varphi_{1}\left(c_{n} ; D\right)+\varphi\left(c_{n} ; D\right) g_{12} \\
p_{123}= & g_{1} \varphi_{23}\left(c_{n} ; D\right)+g_{2} \varphi_{13}\left(c_{n} ; D\right)+g_{3} \varphi_{12}\left(c_{n} ; D\right) \\
& +\varphi_{3}\left(c_{n} ; D\right) g_{12}+\varphi_{2}\left(c_{n} ; D\right) g_{13}+\varphi_{1}\left(c_{n} ; D\right) g_{23} \\
& +g \varphi_{123}\left(c_{n} ; D\right)+g_{123} \varphi\left(c_{n} ; D\right)
\end{aligned}
$$

\section{Appendix 3: Sample sizes}


Table 5: Sample sizes: Adults 25 years old or older

\begin{tabular}{lc}
\hline & Sample size \\
Austria & 11,780 \\
Belgium & 10,765 \\
Croatia & 7,076 \\
Czech & 17,108 \\
Germany & 23,484 \\
Denmark & 10,092 \\
Estonia & 9,518 \\
Spain & 25,348 \\
Finland & 18,402 \\
France & 17,547 \\
Greece & 11,074 \\
Hungary & 16,232 \\
Ireland & 9,478 \\
Iceland & 5,302 \\
Italy & 40,245 \\
Lithuania & 9,365 \\
Luxembourg & 6,978 \\
Latvia & 7,947 \\
Netherlands & 17,601 \\
Norway & 9,835 \\
Poland & 29,294 \\
Portugal & 8,773 \\
Sweden & 11,955 \\
Slovenia & 20,736 \\
Slovakia & 10,262 \\
UK & 15,504 \\
\hline
\end{tabular}

